\title{
Grundsätzliche Erheblichkeit - Kritische Würdigung und mögliche Korrektive
}

\author{
Daniel Emch / Anna Gottret *
}

Das Gaba-Urteil des Bundesgerichts vom 28. Juni 2016 brachte eine wesentliche Neuerung im schweizerischen Kartellrecht. Gewisse Wettbewerbsabreden gelten seither alleine aufgrund ihres Gegenstandes als erheblich und damit als unzulässig, sofern nicht ausnahmsweise eine Effizienzrechtfertigung gelingt. Eine einzelfallweise Erheblichkeitsprüfung anhand quantitativer Kriterien soll bei derartigen Wettbewerbsabreden anders als bis anhin nicht mehr durchgeführt werden. Dieser konzeptionelle Ansatz entspricht u.E. nicht dem Willen des Gesetzgebers. Mit einer Rückkehr zur alten Praxis ist jedoch nicht zu rechnen. Stattdessen ist das Augenmerk auf eine massvolle Umsetzung des neuen Konzepts und auf erforderliche Korrekturen bei der Anwendung anderer Tatbestandsmerkmale zu richten. Die Einführung einer Bagatellschwelle, eine sorgfältige Prüfung der Tatbestandsmerkmale von Art. 4 Abs. 1 KG in Anlehnung an die EU-Praxis, eine restriktive Auslegung der Vermutungstatbestände gemäss Art. 5 Abs. 3 und 4 KG sowie eine wohlwollendere Handhabung der Rechtfertigungsmöglichkeiten würden die über das Ziel hinausschiessende Schärfe des neuen Konzepts zumindest relativieren.

I. Einleitung. 375

II. Konzept der grundsätzlichen Erheblichkeit entspricht nicht dem

Willen des Gesetzgebers

III. Mögliche Korrektive

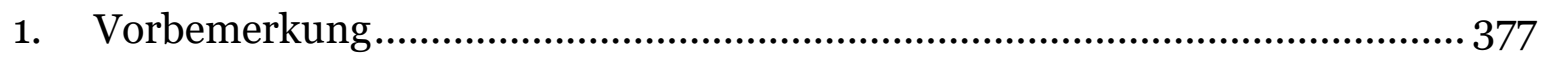

2. Einführung einer Bagatellschwelle ............................................................. 377

3. Sorgfältigere Prüfung von Art. 4 Abs. 1 KG .............................................. 378

4. Restriktivere Auslegung der Vermutungstatbestände..................................379

5. Grosszügigere Handhabung der Rechtfertigungsmöglichkeit .....................380

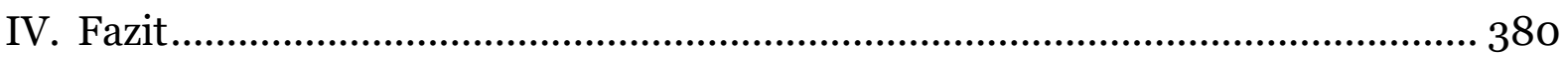

Zitiervorschlag: Daniel Emch/Anna Gottret, Grundsätzliche Erheblichkeit - Kritische Würdigung und mögliche Korrektive, in: sui-generis 2018, S. 374

URL: $\quad$ sui-generis.ch/81

DOI: $\quad$ https://doi.org/10.21257/sg.81

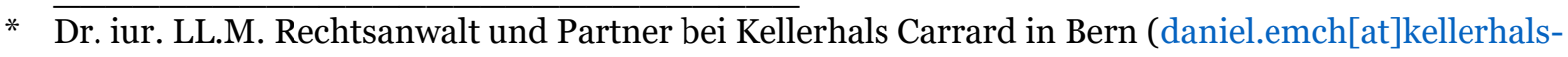
carrard.ch). Dr. iur. LL.M. Rechtsanwältin und Senior Associate bei Kellerhals Carrard in Bern (anna.gottret[at]kellerhals-carrard.ch).

Dieses Werk ist lizenziert unter einer Creative Commons Namensnennung - Weitergabe unter gleichen Bedingungen 4.0 International Lizenz. 


\section{Einleitung}

1 Das Gaba-Urteil1 des Bundesgerichts hat in der Rechtslehre und -praxis hohe Wellen geworfen. Gemäss dem Urteil sollen Wettbewerbsabreden, die unter die Vermutungstatbestände von Art. 5 Abs. 3 und $4 \mathrm{KG}^{2}$ fallen (d.h. zum einen alle horizontalen Abreden über Preise, Mengen und die Aufteilung von Gebieten und Kunden und zum anderen alle vertikalen Abreden über absoluten Gebietsschutz oder Preisbindungen zweiter Hand) neu in jedem Fall zumindest eine erhebliche Beeinträchtigung des wirksamen Wettbewerbs im Sinne von Art. 5 Abs. 1 KG darstellen und mit direkten Sanktionen geahndet werden können, wenn sie sich nicht ausnahmsweise durch Gründe der wirtschaftlichen Effizienz rechtfertigen lassen. Mit anderen Worten soll von einer «grundsätzlichen» oder per-seErheblichkeit aufgrund des Gegenstandes der Abrede ausgegangen werden. Eine einzelfallweise Erheblichkeitsprüfung anhand quantitativer Kriterien soll bei solchen Wettbewerbsabreden abweichend von der früheren Praxis nicht mehr erforderlich sein.

Der vorliegende Beitrag enthält vorab einige kritische Gedanken zu dieser Praxisänderung. Mithin wird aufgezeigt, dass die vom Bundesgericht mit dem Gaba-Urteil neu eingeführte per-seErheblichkeit gewisser Wettbewerbsabreden nicht dem Willen des Gesetzgebers entsprochen haben dürfte (Ziff. II). Zugleich soll der Beitrag konkrete Vorschläge für einen möglichen künftigen Umgang mit dem Konzept der grundsätzli-

\footnotetext{
BGE 143 II 297.
}

Bundesgesetz über Kartelle und andere Wettbewerbsbeschränkungen vom 6. Oktober 1995 (Kartellgesetz, KG; SR 251). chen Erheblichkeit liefern. Schliesslich muss angesichts der expliziten Bestätigung des Konzepts in drei weiteren Urteilen (BMW3, Baubeschläge4, Altimum5) doch damit gerechnet werden, dass das Bundesgericht in den nächsten Jahren daran festhalten und nicht zur alten Praxis zurückkehren wird. Insbesondere sollen mögliche Korrektive zu dieser u.E. zu rigid ausgefallenen Auslegung des Erheblichkeitsbegriffs aufgezeigt werden (Ziff. III). Abgerundet wird der Beitrag schliesslich durch ein Schlussfazit (Ziff. IV).

\section{Konzept der grundsätzlichen Erheblichkeit entspricht nicht dem Willen des Gesetzgebers}

3 Mit dem Kartellgesetz 95 wollte das Parlament eine Missbrauchsgesetzgebung erlassen. Interventionen sollten nur dann erfolgen, wenn eine Verhaltensweise erhebliche Auswirkungen auf den wirksamen Wettbewerb hat. Das belegt unter anderem die folgenden Passage aus der Botschaft 1994\%:

«Massgebend ist, ob die Auswirkungen einer Wettbewerbsbeschränkung volkswirtschaftlich oder sozial schädlich sind. Nur wenn die Schädlichkeit im Einzelfall festgestellt wurde, ist die Wettbewerbsbeschränkung unzulässig.»

3 Urteil des Bundesgerichts 2C_63/2016 vom 24. Oktober 2017.

4 Urteil des Bundesgerichts 2C_1016/2014 vom 9. Oktober 2017.

5 Urteil des Bundesgerichts 2C_101/2016 vom 18. Mai 2018.

6 Botschaft zu einem Bundesgesetz über Kartelle und andere Wettbewerbsbeschränkungen (Kartellgesetz, KG) vom 23. November 1994 (BBl 1995 I 468), S. $554 \mathrm{f}$. 
4 Zugleich sollte - wie die folgende weitere Passage aus der Botschaft 1994 illustriert - nach dem Willen des Parlaments explizit am bereits im Kartellgesetz 1985 enthaltenen Erheblichkeitskriterium und der dazu entwickelten Praxis festgehalten werden:

«Da bereits das geltende Kartellgesetz [i.e. Kartellgesetz von 1985] die Erheblichkeit als Voraussetzung für die Anwendbarkeit der materiellen Kartellbestimmung nennt, kann auf die entsprechende Praxis verwiesen werden, in welcher versucht wird, dem Begriff der Erheblichkeit Kontur zu verleihen.»7

5 Das Kartellgesetz $1985^{8}$ hatte zwischen einem zivilrechtlichen (subjektiven) und einem verwaltungsrechtlichen (objektiven) Erheblichkeitsbegriff differenziert.9 Gemäss der damaligen Rechtsprechung war eine Behinderung «erheblich», wenn sie eine gewisse Intensität aufwies und von den Betroffenen als solche empfunden wurde. ${ }^{10}$ Somit war nicht die Art der Wettbewerbsbehinderung entscheidend, sondern deren Auswirkung auf das wirtschaftliche Verhalten des Betroffenen.

6 Ebenso klar aus den Materialien herauszulesen ist ferner, dass nach dem Willen des Gesetzgebers für den Fall dass eine Vermutung widerlegt werden kann, die Wettbewerbsabrede daraufhin zu überprüfen sei, ob sie eine erhebliche Wett-

BBl 1995 I 468 (Fn. 6), S. 554.

8 Bundesgesetz vom 20. Dezember 1985 über Kartelle und ähnliche Organisationen (Kartellgesetz, KG), aufgehoben am 1. Februar 1996.

9 Krauskopf/Schaller, Art. 5 KG in: Basler Kommentar, Kartellgesetz, 1. Aufl. 2010, N. 152.

10 BGE 112 II 268 E. I.2.b S. 276. bewerbsbeeinträchtigung verursache.11 Die Botschaft 1995 führt hierzu zwar aus, dass dies in der Regel der Fall sein dürfte. ${ }^{12}$ Von einer Überprüfung der Auswirkungen im Einzelfall dispensiert sie jedoch nicht. Eine Erheblichkeitsfiktion war vom Gesetzgeber nicht vorgesehen. Das anerkannte ursprünglich auch das Bundesgericht, wies es im Fall Sammelrevers ${ }^{13}$ doch beispielsweise noch explizit darauf hin, dass nach Widerlegung der Vermutung eine erhebliche Wettbewerbsbeeinträchtigung vorliege, sofern die Abrede Güter mit einem wesentlichen Marktanteil betreffe. ${ }^{14}$

7 Das Gaba-Urteil weicht diametral von dieser früheren Rechtsprechung ab, ohne dass das Bundesgericht die Praxisänderung in seinen Erwägungen offenlegt und ohne dass dafür ein äusserer Anlass auszumachen ist. Mithin hat sich die massgebliche Rechtslage seit dem Urteil im Fall Sammelrevers nicht geändert. Die kurz nach diesem Urteil in Kraft gesetzte KG-Revision 2003 beinhaltete keinerlei Anpassungen der Konzeption des Erheblichkeitsbegriffs. Weder in der Botschaft ${ }^{15}$ noch in den parlamentarischen Beratungen ${ }^{16}$ findet sich ein Hinweis, wonach das Erheblichkeitskriterium in Vermutungsfällen gänzlich aufgehoben werden sollte. Die Beratungen im Ständerat und im Nationalrat waren kurz und knapp. Der zitierte Bundesgerichtsentscheid zum Sammelrevers lag damals vor. In keiner Wortmeldung sind An-

BBl 1995 I 468, S. 565 f.

12 BBl 1995 I 468, S. 566.

BGE 129 II 18.

14 BGE 129 II 18 E. 5.2.2 S. 25.

15 Botschaft über die Änderung des Kartellgesetzes vom 7. November 2001 (BBl 2001 2022).

16 Geschäft des Bundesrates (01.071): Kartellgesetz. Änderung, Ratsdebatte Nationalrat, Herbstsession 2002, fünfte Sitzung, 19. September 2002. 
haltspunkte dafür zu finden, dass eine Praxisänderung bei den Horizontalabreden beabsichtigt worden war. Es gibt auch keine Aussage, wonach in Vertikalfällen auf das Kriterium der Erheblichkeit verzichtet werden sollte.

Vielmehr führt die Botschaft aus dem Jahr 2001 explizit aus, dass harte Abreden nicht direkt sanktioniert werden können, wenn sie den wirksamen Wettbewerb weder beseitigen noch erheblich beeinträchtigen: ${ }^{17}$

«Vom Anwendungsbereich der direkten Sanktionen ausgenommen sind demnach nach Artikel 5 Absatz 1 unzulässige Verhaltensweisen, welche keine Preis-, Mengen- oder Gebietsabreden zum Gegenstand haben. Ebenso wenig können Preis-, Mengen- und Gebietsabreden direkt sanktioniert werden, die den wirksamen Wettbewerb weder beseitigen noch erheblich beeinträchtigen beziehungsweise die durch Gründe der wirtschaftlichen Effizienz gerechtfertigt sind.»

Auch mit dieser Botschaftsstelle steht die vom Bundesgericht mit dem Gaba-Urteil neu eingeführte per-se-Erheblichkeit in Widerspruch, kommt hier doch deutlich zum Ausdruck, dass es nach Auffassung des Gesetzgebers durchaus Konstellationen gibt, in denen eine Abrede einen Vermutungstatbestand zwar erfüllt, aber nicht erheblich ist.

\section{Mögliche Korrektive}

\section{Vorbemerkung}

10 Das vom Bundesgericht mit dem GabaUrteil neu eingeführte Konzept der grundsätzlichen Erheblichkeit birgt die Gefahr, dass inskünftig auch Abreden und Verhaltensweisen in Konflikt mit dem Kartellrecht geraten könnten, die bei Lichte betrachtet keine spürbaren Auswirkungen auf den Wettbewerb haben. Dieser Gefahr muss nach Auffassung der Autoren dieses Beitrags mit einer massvollen Umsetzung des Konzepts der grundsätzlichen Erheblichkeit und Praxispräzisierungen bei anderen Tatbestandsmerkmalen begegnet werden. Insbesondere sollten die nachstehend aufgeführten Korrektive ins Auge gefasst werden.

\section{Einführung einer Bagatellschwelle}

11 In der mündlichen Urteilsberatung zum Gaba-Urteil war von einer Bagatellschwelle die Rede. Die Bundesrichter hielten dafür, dass Absprachen zwischen zwei Schreinern im Sinne einer Bagatelle auch weiterhin noch zulässig sein bzw. nicht aufgegriffen werden sollten. In der schriftlichen Urteilsbegründung findet sich diese Bagatellschwelle zwar nicht mehr explizit. Trotzdem sollte dieser mögliche Ansatz zur sachgerechten Eingrenzung der grundsätzlichen Erheblichkeit aufgenommen und weiterverfolgt werden. Kleine und unbedeutende Unternehmen sollten sich nicht mit den schwierigen Abgrenzungsfragen von Art. $5 \mathrm{KG}$ auseinandersetzen müssen. ${ }^{18}$

18 Bspw. die Fragen, wann eine Preisabrede vorliegt oder wann genau ein Informationsaustausch als Wettbewerbsabrede qualifiziert werden kann, sind in der Praxis nicht leicht und nur unter Beizug von teuren Experten zu beantworten. 
Sie haben weder die personellen noch die finanziellen Ressourcen dazu. Die GabaRechtsprechung lässt durchaus Freiraum für eine Bagatellklausel. Da die Rechtsprechung bloss «grundsätzlich» und nicht generell von einer Erheblichkeitsfiktion ausgeht, steht es der WEKO und den Gerichten frei, den übrig gebliebenen Auslegungsspielraum mit einer Bagatellklausel auszufüllen. ${ }^{19}$ Die Bagatellklausel müsste im Sinne justiziabler Tatbestandsmerkmale ausgestaltet werden und nicht bloss als Aufgreifkriterium, dessen Anwendung im Ermessen der Wettbewerbsbehörden und Gerichte steht.

Naheliegende Instrumente zur Einführung und Definition einer solchen Bagatellschwelle wären dabei die Bekanntmachung über die wettbewerbsrechtliche Behandlung vertikaler Abreden (Vertikalbekanntmachung, VerkBek) oder die dazugehörigen Erläuterungen der Wettbewerbskommission (VertBek-Erläuterungen). Bislang wurden diese Dokumente jedoch bedauerlicherweise nicht um entsprechende Bestimmungen ergänzt.20 Der WEKO bleibt es zudem unbenommen, in ihrer Praxis ein solches Konzept zu entwickeln und im Einzelfall darauf zu verweisen, dass eine Abrede, welche zwar formal unter einen Vermutungstatbestand von Art. 5 Abs. 3 oder 4 KG fallen würde, aufgrund des Bagatellcharakters im konkreten Fall nicht erheblich sei.

\section{Sorgfältigere Prüfung von Art. 4} Abs. 1 KG

13 Ein weiteres mögliches Korrektiv ist die Anwendung von Art. 4 Abs. 1 KG. Vor dem Gaba-Urteil hat die Frage, ob eine Abrede eine Wettbewerbsbeschränkung bezweckt oder bewirkt, kaum eine Rolle gespielt. ${ }^{21}$ Praxisgemäss musste ohnehin eine Erheblichkeitsprüfung durchgeführt werden. Da Wirkungen nachgewiesen werden mussten, war die Kategorie der bezweckten Wettbewerbsbeschränkung irrelevant. Neu muss Art. 4 Abs. 1 KG sorgfältiger angewendet werden. ${ }^{22}$

14 Insbesondere muss in Analogie zur einschlägigen EU-Rechtsprechung künftig jeweils unter Mitberücksichtigung der wirtschaftlichen und rechtlichen $\mathrm{Zu}$ sammenhänge sowie der Ziele der Parteien in jedem Einzelfall sorgfältiger geprüft werden, ob tatsächlich von einer bezweckten Wettbewerbsbeschränkung ausgegangen werden kann. Wie die EURechtsprechung zeigt, ist dies selbst bei Preisabreden nicht zwingend der Fall. ${ }^{23}$ Gemäss der Carte-Bancaire-Rechtsprechung des EuGH ist im Einzelfall zu begründen, weshalb eine Abrede so schädlich ist, dass sie als «bezweckte» Wettbewerbsbeschränkung betrachtet werden darf; auch in der EU-Praxis kann mit anderen Worten nicht einfach behauptet werden, eine gewisse Abredeform sei grundsätzlich schädlich, es müssen im Einzelfall vielmehr die Marktstruktur und die darauf bestehenden tatsächli-

21 Vgl. Baldi (Fn. 19), S. 70.

22 So auch Gübeli Raffael, Unterschiede in der Qualifikation von horizontal und vertikal abgestimmten Verhaltensweisen in der Praxis, in: sic! 2018 S. 229 - 241, 229, oder Baldi (Fn. 19), S. 79.

23 Urteil des EuGH C-67/13 P vom 11. September 2014, CB/Kommission Rz. 53. 
chen Bedingungen analysiert werden. ${ }^{24}$. Diese Grundsätze müssen zwingend auch bei der Anwendung des Schweizer Kartellgesetzes berücksichtigt werden.25. Wenn bei Vorliegen eines Vermutungstatbestandes automatisch eine «bezweckte Wettbewerbsbeschränkung» angenommen würde, würde das entsprechende Tatbestandselement von Art. 4 Abs. 1 KG seines Sinnes entleert.

\section{Restriktivere Auslegung der Vermutungstatbestände}

15 Die Wettbewerbskommission hat die Vermutungstatbestände von Art. 5 Abs. 3 und $4 \mathrm{KG}$ sehr weit ausgelegt. ${ }^{26}$ Bislang wurden bspw. alle Abreden, welche einen irgendwie gearteten Bezug zu Preisen, Preisbestandteilen oder Preiselementen hatten, als Preisabreden im Sinne von Art. 5 Abs. 3 lit. a KG qualifiziert. ${ }^{27}$ So wurden bspw. auch Abreden über Bruttopreise als Preisabreden qualifiziert, obgleich die an der «Bruttopreisabrede» beteiligten Unternehmen sich harten Rabattwettbewerb geleistet haben. ${ }^{28}$

Die Erheblichkeitshürde bot bislang ein gewisses Korrektiv zur äusserst weiten Auslegung von Art. 5 Abs. 3 und 4 KG durch die Wettbewerbsbehörden. Da die-

24 Urteil des EuGH C-67/13 P vom 11. September 2014, CB/Kommission Rz. 69, vgl. auch Heinemann Andreas, Die Erheblichkeit bezweckter und bewirkter Wettbewerbsbeschränkungen, in: Jusletter vom 29. Juni 2015, Rz. 35.

25 Vgl. Heinemann Andreas, Das Gaba-Urteil des Bundesgerichts: Ein Meilenstein des Kartellrechts, in: ZSR 2018 I, S. 118.

26 Vgl. Heinemann (Fn. 25), S. 110.

27 So auch Tuchschmid Felix, Die horizontale Preisabrede - Eine Auslegung des kartellrechtlichen Vermutungstatbestands von Art. 5 Abs. 3 lit. a KG, AJP 2018, S. 848 und Baldi Marino, «Zweimal hü und zweimal hott» beim Schweizer Kartellgericht, in: AJP 2016 S. 315-322, 321.

28 Verfügung der WEKO vom 29. Juni 2015 i.S. Badezimmer, Rz. 2444 ff. (noch nicht publiziert). ses Korrektiv nun weggefallen ist, tut eine kritische Überprüfung der bisherigen Praxis zu diesem Punkt not. Dabei ist zu berücksichtigen, dass kartellrechtliche Sanktionen einen strafrechtsähnlichen Charakter haben und die Verfahrensgarantien der EMRK in kartellrechtlichen Sanktionsverfahren anwendbar sind. ${ }^{29}$ Entsprechend sind die sanktionsbegründenden Vermutungstatbestände von Art. 5 Abs. 3 und 4 KG gemäss dem Bestimmtheitsgebot bzw. dem Grundsatz nulla poena sine lege certa eng auszulegen. 30

17 Insbesondere sollte künftig nicht mehr alles, was einen entfernten Bezug zum Wettbewerbsparameter Preis hat, als horizontale Preisfestsetzung oder als Preisbindung zweiter Hand qualifiziert werden. ${ }^{31}$ Nur Abreden über Preiskomponenten, die tatsächlich zu einer einheitlichen Preisfestsetzung führen, gehören unter die betreffenden Vermutungstatbestände subsumiert. Dieselbe Problematik besteht auch beim Tatbestand des absoluten Gebietsschutzes nach Art. 5 Abs. 4 KG: Bei Fällen nach Art. 5 Abs. 4 KG muss nachgewiesen werden, dass einem ausländischen Distributor Passivverkäufe in das Gebiet der Schweiz untersagt worden sind. Ein Vertrag zwischen einem ausländischen Lieferanten und einem Schweizer Vertriebspartner, wonach sich der Lieferant bemühe, Querlieferungen in die Schweiz zu verhindern, genügt entgegen der Praxis der WEKO - nicht. ${ }^{2}$

29 BGE 139 I 72 E.2.2 S. 78.

30 Zur künftigen Auslegung von Art. 5 Abs. 3 und 4 KG vgl. Birkhäuser Nicolas/Reinert Mani, Das Gaba-Urteil des Bundesgerichts: Kritik und künftige Anwendung, in: ZSR 2018 I, S. 132 und 140

31 Vgl. Heinemann (Fn. 25), S. 112.

$3^{2}$ So aber die WEKO in: Verfügung der WEKO vom 27. Mai 2013 i.S. Marché du livre écrit en français, Rz. 207 ff. (noch nicht publiziert). 


\section{Grosszügigere Handhabung der Rechtfertigungsmöglichkeit}

18

Schliesslich würde auch eine grosszügigere Handhabung der in Art. 5 Abs. 2 KG vorgesehenen Möglichkeit, unter die Vermutungstatbestände fallende Wettbewerbsabreden durch Gründe der wirtschaftlichen Effizienz zu rechtfertigen, die Auswirkungen der grundsätzlichen Erheblichkeit etwas entschärfen. Zirkuläre Begründungen, wonach HardcoreAbreden nur gerade in absoluten Ausnahmefällen durch Gründe der wirtschaftlichen Effizienz zu rechtfertigen seien, sind unter den veränderten Rahmenbedingungen nicht mehr haltbar. Die Tatsache, dass die Rechtfertigung durch Gründe der wirtschaftlichen Effizienz neu die einzige Möglichkeit ist, um bei Vorliegen einer unter Art. 5 Abs. 3 oder 4 KG zu subsumierenden Wettbewerbsabrede eine direkte Sanktion abzuwenden, bedingt, dass dieser Punkt künftig detailliert und ergebnisoffen geprüft wird.33

\section{Fazit}

19 Urteil neu eingeführte Konzept der grundsätzlichen Erheblichkeit entspricht u.E. nicht dem Willen des Gesetzgebers. Mit einer Korrektur des betreffenden Urteils durch das Bundesgericht ist jedoch nicht zu rechnen. Stattdessen sind Korrektive in anderen Punkten erforderlich, wie etwa die Einführung einer Bagatellschwelle, eine sorgfältigere Prüfung des Vorliegens einer Wettbewerbsabrede im jeweiligen Einzelfall, eine restriktivere Auslegung der Vermutungstatbestände und/oder eine grosszügigere Handhabung der Möglichkeit, unter Art. 5 Abs. 3

33 So auch Heinemann (Fn. 25), S. 111 und 118. oder 4 KG fallende Wettbewerbsabreden durch Gründe der wirtschaftlichen Effizienz zu rechtfertigen.

20 Zugleich wäre wünschbar, dass sich sowohl die WEKO als auch die Gerichte zum Grundsatz bekennen, dass was in der EU erlaubt ist, auch in der Schweiz erlaubt sein muss. Als es um die Einführung des Teilkartellverbotes bei der letzten, gescheiterten KG-Revision34 ging, hat der Bundesrat den Erlass einer entsprechenden Verordnung im Sinne von Art. $6 \mathrm{KG}$ in Aussicht gestellt. Damit sollte die von gewissen Parlamentariern befürchtete Härte des Teilkartellverbots mit Rechtfertigungsmöglichkeiten relativiert werden. Angesichts der durch das GabaUrteil ausgelösten Unsicherheiten wäre zu begrüssen, wenn sich das SECO erneut Gedanken über dieses Instrument machen würde. Mangels Präzedenzfälle aus der Schweiz orientieren sich die Unternehmen nämlich an den EUGruppenfreistellungsverordnungen und den entsprechenden Guidelines. Es fehlt aber die erforderliche Rechtssicherheit, dass ein solches Vorgehen vom Bundesgericht geschützt würde. Die Ausführungen im Gaba-Urteil zur Nichtanwendbarkeit der Technologietransfer-GVO35 lassen nicht auf eine entsprechende Grundhaltung des Bundesgerichts schliessen. In diesem Zusammenhang könnte eine Verordnung des Bundesrates die erforderliche Rechtssicherheit schaffen. Rechtssicherheit ist für die Unternehmen absolut zentral.

\footnotetext{
$34 \overline{\text { Geschäft des Bundesrats (12.028): Kartellgesetz. }}$ Änderung.
}

35 BGE 143 II 297 E. 6.4.1 S. $331 \mathrm{f}$. 